\title{
A New Family of Optical Codes Based on Complementary Theory for OCDMA System*
}

\author{
Xing Chen, Weixiao Meng \\ School of Electronics and Information Engineering, Harbin Institute of Technology, Harbin, China \\ Email: xingchen@hit.edu.cn,wxmeng@hit.edu.cn
}

Received June, 2013

\begin{abstract}
A new family of optical codes for Optical Code-division Multiple Access (OCDMA) systems, named as Optical Complementary Codes (OCCs), is proposed in this paper. The constructions of these codes consist of multiple sub-codes, and the codes have an auto-correlation interference constraint as 0 and a cross-correlation interference constraint as 1. Compared with conventional optical codes such as OPCs, OOCs and 2-D OOCs, the OCC has a shorter code length and higher code efficiency with better correlation property.
\end{abstract}

Keywords: Optical Code-division Multiple Access (OCDMA); Complementary Code

\section{Introduction}

Optical Code Division Multiple Access (OCDMA) introduces CDMA to optical communication system and combines the flexibility of CDMA system and the broadband of fibre communication system. Compared with Wavelength-division Multiplexing (WDM) and Time-division Multiplexing (TDM), OCDMA shares the frequency resources and time resources with all the users by signature codes. It is the only way to achieve an asynchronous and decentralized network with the whole bandwidth utilized by each user [1]. Besides, the OCDMA system shares the same security potential with the radio frequency CDMA system because they have a similar spectrum spreading method.

Optical CDMA is a suitable access method to achieve random access, high capacity and contention free especially for busty traffic. Besides, OCDMA can be efficiently used with WDM and TDM in practical system to satisfy different traffic requirements and multiple services. For now, OCDMA is an important method for achieving all-optical networks.

Optical codes for OCDMA are totally different from codes for the CDMA systems in wireless communications, and the most important difference is that there is no negative signal in optical transmission. Although some bipolar OCDMA systems have been proposed, they are not well received by the majority because of the high cost and complexity in implementation [2]. Hence, the optical codes for OCDMA are unipolar codes mainly,

*This Article is sponsored by National Science and Technology Major Project (2012ZX03004003) and in this article, we will discuss the unipolar optical codes only. Since finding a proper optical code set is a very important issue in OCDMA, this paper will propose a new family of optical codes achieving optimum correlation properties, named as optical complementary codes (OCCs).

The rest of this paper is organized as follows. In the next section, the theory of complementary codes is introduced first, and then the characteristics of optical codes which have ideal correlation properties are deduced. In section III, the construction method of optical complementary coding is proposed with examples. Section IV discusses the correlation properties and capacities of OCCs. Section V concludes this paper.

\section{Fundamental Theories Involved in OCCs}

\subsection{Complementary Codes}

The concept of complementary codes is introduced firstly in 1949 by Golay with the name as Complementary Series [3]. In 1961, he proposed the theory and a construction method for Complementary Series [4]. The series proposed by Colay is constructed with a pair of binary sequences in the same length, and he calls each series sub-code. The auto-correlation and cross-correlation are different from those of traditional CDMA signature codes. Let $\boldsymbol{a}$ and $\boldsymbol{b}$ denote two complementary sequences where $\boldsymbol{a}=\left\{\boldsymbol{a}_{1}, \boldsymbol{a}_{2}\right\}$ and $\boldsymbol{b}=\left\{\boldsymbol{b}_{1}, \boldsymbol{b}_{2}\right\}, \boldsymbol{a} \neq \boldsymbol{b}$. The operation of complementary correlations is a little complex than the conventional one. In complementary correlations, each sub-codes correlate with each other correspondingly and then the results will be summed together. This opera- 
tion is described as follows:

the complementary auto-correlation is denoted as

$$
\boldsymbol{a} \bullet \boldsymbol{a}=\boldsymbol{a}_{1} \cdot \boldsymbol{a}_{1}+\boldsymbol{a}_{2} \bullet \boldsymbol{a}_{2}
$$

the complementary cross-correlation is denoted as

$$
\boldsymbol{a} \cdot \boldsymbol{b}=\boldsymbol{a}_{1} \cdot \boldsymbol{b}_{1}+\boldsymbol{a}_{2} \cdot \boldsymbol{b}_{2}
$$

where the symbol - means the inner product.

In 2001, Hsiao-Hwa Chen evolved the complementary pairs into complementary codes with multiple sub-codes which could achieve better orthogonality. These codes, called orthogonal complete complementary codes [5], decreased the MAI (Multi Access Interference) of CDMA systems with simple code constructions. In this paper, we will construct OCCs using a similar method as the work of [5]

\subsection{Characteristics of Optical Codes with Ideal Complementary Propertie}

Even though the OCDMA is introduced from CDMA system in wireless communication and share a similar construction with it, the codes for OCDMA is quite different from those in CDMA. In fibre communication system, the data are encoded with binary codes, which are 0 s and 1s, while the codes in CDMA are bipolar code described as $-1 \mathrm{~s}$ and $+1 \mathrm{~s}$. So, it is impossible to achieve a perfect orthogonal property. Though some of the researchers have proposed some systems with bipolar codes, they are not well received because of their complexity and skyscraping cost. With the discussion of binary codes in optical CDMA systems.

Since the code length and code weight will influence the properties of optical codes, however, to the best of our knowledge, there is no research on the characteristics of optical codes with ideal auto- and cross- correlation has been published. Meanwhile, it is very important for this paper to introduce the optical complementary codes, so it is reasonable to deduce the characteristics. Besides, the optical complementary codes are based on these derivations.

The codes in OCDMA systems are presented with their parameters. To a specific set of optical codes, it is described as $\left(n, w, \lambda_{a}, \lambda_{c}\right)$, where $n$ is the length of the codes, $w$ is the hamming weight of a code, $\lambda_{a}$ and $\lambda_{c}$ are the constrains of auto-correlation and cross-correlation respectively. Let code set $\boldsymbol{C}$ denote $\left(n, w, \lambda_{a}, \lambda_{c}\right)$, $\boldsymbol{a}, \boldsymbol{b} \in \boldsymbol{C} \quad$ and $\boldsymbol{a}=\left\{a_{1}, a_{2}, \cdots, a_{n}\right\}, \quad \boldsymbol{b}=\left\{b_{1}, b_{2}, \cdots, b_{n}\right\}$, $\boldsymbol{a} \neq \boldsymbol{b}$. Then, the code set must satisfy the follows:

$$
\begin{aligned}
& \lambda_{a} \geq \sum_{i=1}^{n} a_{i} \times a_{i \oplus_{n} r} \text { where } r>0 \\
& \lambda_{c} \geq \sum_{i=1}^{n} a_{i} \times b_{\oplus_{n} r} \text { where } r \geq 0
\end{aligned}
$$

In these equations $r$ is any integer which denotes the shift of code chips and $\oplus_{n}$ means the addition with modulo $n$.

MAI is the most important interference in OCDMA system [6]. In order to minimize the MAI, the best way is to design a new family of codes with better correlation properties. Furthermore, the OCDMA systems are inherently chip-asynchronous because the users access the systems randomly without coordination [7], so it is important for codes to achieve a lower auto-correlation constrains.

Let $\boldsymbol{C}$ denote a one dimensional optical code set $\left(n, w, \lambda_{a}, \lambda_{c}\right), \quad \boldsymbol{a}, \boldsymbol{b} \in \boldsymbol{C}, \boldsymbol{a}=\left\{a_{1}, a_{2}, \cdots, a_{n}\right\}, \quad \boldsymbol{b}=\left\{b_{1}, b_{2}, \cdots, b_{n}\right\}$, $\boldsymbol{a} \neq \boldsymbol{b} . \boldsymbol{a}^{\tau}$ is the shifted sequence $\boldsymbol{a}$ with $\tau$ shifts, and $\boldsymbol{b}^{(\tau)}$ is the shifted sequence $\boldsymbol{b}$ with $\tau$ shifts. $S_{a, \tau}$ is the auto- correlation value of $\boldsymbol{a}$ and $\boldsymbol{a}^{(\tau)}, S_{c, \tau}$ is the cross-correlation value of $\boldsymbol{a}$ and $\boldsymbol{b}^{(\tau)} . S_{a}$ and $S_{c}$ are the sum of $S_{a, \tau}$ and $S_{c, \tau}$ respectively. Hence, we could get

$$
\begin{aligned}
S_{a, \tau} & =\boldsymbol{a} \bullet \boldsymbol{a}^{(\tau)} \\
S_{c, \tau} & =\boldsymbol{a} \cdot \boldsymbol{b}^{(\tau)}
\end{aligned}
$$

It can be also described as

$$
\begin{aligned}
& S_{a, 0}=a_{1} a_{1}+a_{2} a_{2}+\cdots+a_{n-1} a_{n-1}+a_{n} a_{n} \\
& S_{a, 1}=a_{1} a_{2}+a_{2} a_{3}+\cdots+a_{n-1} a_{n}+a_{n} a_{1} \\
& S_{a, 2}=a_{1} a_{3}+a_{2} a_{4}+\cdots+a_{n-1} a_{1}+a_{n} a_{2} \\
& \vdots \\
& S_{a, n-1}=a_{1} a_{n}+a_{2} a_{1}+\cdots+a_{n-1} a_{n-2}+a_{n} a_{n-1}
\end{aligned}
$$

Then, we can get

$$
S_{a}=\sum_{i=0}^{n-1} S_{a, i}=\sum_{i=0}^{n-1} a_{i}\left(\sum_{j=0}^{n-1} a_{j}\right)=\sum_{i=0}^{n-1} a_{i} \sum_{j=0}^{n-1} a_{j}=w^{2}
$$

When the shift is 0 , the auto-correlation reaches its peek, and this condition dose not consist interference. Then, we can get the sum of auto-correlation interference as

$$
w^{2}-w=w(w-1)
$$

In a similar way, we can get

$$
S_{c}=\sum_{i=0}^{n-1} S_{c, i}=\sum_{i=0}^{n-1} a_{i}\left(\sum_{j=0}^{n-1} b_{j}\right)=\sum_{i=0}^{n-1} a_{i} \sum_{j=0}^{n-1} b_{j}=w^{2}
$$

and the sum of cross-interference is $w^{2}$.

It could be concluded that, with the increase of code weight $w$, the sum of auto-correlation and the sum crosscorrelation both increase sharply. In order to achieve the minimum of interference and the best of correlation properties, the code weight should be 1 . Then the sum of auto-correlation is 0 and $S_{c}=1$, and we could get each auto-correlation interference is 0 and the maximum of cross-correlation is 1 , i.e. correlation constraints are $\lambda_{a}=0$ and $\lambda_{c}=1$.

But the auto-correlation peek of this kind of one dimension codes is 1 , which could not be applied into practical OCDMA system. In order to achieve a lager 
auto-correlation peek, the construction of multiple subcodes will be introduced into OCC.

Let $m$ be the length of a subcode, $n$ be the quantity of sub-codes in one code. Each subcode shares a weight as $w$, so the code weight is $n w$, the auto-correlation constrain is $\lambda_{a}$ and cross-correlation constrain is $\lambda_{c}$, code $\boldsymbol{a}, \boldsymbol{b}$ presented as

$$
\begin{aligned}
& \boldsymbol{a}=\left\{\left[\boldsymbol{a}_{1}\right],\left[\boldsymbol{a}_{2}\right], \cdots,\left[\boldsymbol{a}_{n}\right]\right\} \\
& \boldsymbol{b}=\left\{\left[\boldsymbol{b}_{1}\right],\left[\boldsymbol{b}_{2}\right], \cdots,\left[\boldsymbol{b}_{n}\right]\right\}
\end{aligned}
$$

This kind of code could be regarded a two dimensional code. We could reconstruct it into the form of conventional 2-D optical codes such as 2-D OOC ( two dimensional optical orthogonal codes). The codes described above could be reformed into a $n \times m$ matrix, and each row of the matrix is a subcode. So the codes could be reformed as

$$
\begin{gathered}
\boldsymbol{a}=\left[\begin{array}{c}
\boldsymbol{a}_{1} \\
\boldsymbol{a}_{2} \\
\vdots \\
\boldsymbol{a}_{n}
\end{array}\right]=\left[\begin{array}{cccc}
a_{11} & a_{12} & \cdots & a_{1 m} \\
a_{21} & a_{21} & \cdots & a_{2 m} \\
\vdots & \ddots & & \vdots \\
a_{n 1} & a_{n 2} & \cdots & a_{n m}
\end{array}\right] \\
\boldsymbol{b}=\left[\begin{array}{c}
\boldsymbol{b}_{1} \\
\boldsymbol{b}_{2} \\
\vdots \\
\boldsymbol{b}_{n}
\end{array}\right]=\left[\begin{array}{cccc}
b_{11} & b_{12} & \cdots & b_{1 m} \\
b_{21} & b_{21} & \cdots & b_{2 m} \\
\vdots & \ddots & & \vdots \\
b_{n 1} & b_{n 2} & \cdots & b_{n m}
\end{array}\right]
\end{gathered}
$$

The constraints of correlations in two dimensional codes is similar with the one dimensional one,

$$
\begin{aligned}
& \lambda_{a} \geq \sum_{i=1}^{n} \sum_{j=1}^{m} a_{i, j} \times a_{i, j \oplus_{n} r} \text { where } r>0 \\
& \lambda_{c} \geq \sum_{i=1}^{n} \sum_{j=1}^{m} a_{i, j} \times b_{i, j \oplus_{n} r} \text { where } r \geq 0
\end{aligned}
$$

In these equations $r$ is any integer which denotes the shift of code chips and $\oplus_{n}$ means the addition with modulo $n$.

The codes with shift $\tau$ are $\boldsymbol{a}^{\tau}$ and $\boldsymbol{b}^{\tau}$. The autocorrelation peek for each subcode is $w$, so $\boldsymbol{a} \bullet \boldsymbol{a}=n w$, and we could get

$$
\begin{gathered}
S_{a}=\sum_{\tau=0}^{m-1} \boldsymbol{a} \cdot \boldsymbol{a}^{\tau}=\sum_{\tau=0}^{m-1}\left[\begin{array}{c}
\boldsymbol{a}_{1} \\
\boldsymbol{a}_{2} \\
\vdots \\
\boldsymbol{a}_{n}
\end{array}\right]\left[\begin{array}{c}
\boldsymbol{a}_{1}^{\tau} \\
\boldsymbol{a}_{2}^{\tau} \\
\vdots \\
\boldsymbol{a}_{n}^{\tau}
\end{array}\right]^{\mathrm{T}} \\
=\left[\begin{array}{c}
\boldsymbol{a}_{1} \\
\boldsymbol{a}_{2} \\
\vdots \\
\boldsymbol{a}_{n}
\end{array}\right] \sum_{\tau=0}^{m-1}\left[\begin{array}{c}
\boldsymbol{a}_{1}^{\tau} \\
\boldsymbol{a}_{2}^{\tau} \\
\vdots \\
\boldsymbol{a}_{n}^{\tau}
\end{array}\right]^{\mathrm{T}}\left[\begin{array}{c}
\boldsymbol{a}_{1} \\
\boldsymbol{a}_{2} \\
\vdots \\
\boldsymbol{a}_{n}
\end{array}\right]^{\mathrm{T}}\left[\begin{array}{c}
\boldsymbol{a}_{1}^{\tau} \\
\boldsymbol{a}_{2}^{\tau} \\
\vdots \\
\boldsymbol{a}_{n}^{\tau}
\end{array}\right]^{\mathrm{T}}=n w^{2} \\
S_{c}=\sum_{\tau=0}^{m-1} \boldsymbol{a} \cdot \boldsymbol{b}^{\tau}=n w^{2}
\end{gathered}
$$

Where “•" is the inner product and $[\cdot]^{\mathrm{T}}$ is the transpose of a matrix. Similarly, we could get the minimums of auto- correlation sum and cross-correlation sum when $w$ is 1 . When $w=1$, the sum of auto-correlation interferences is $n w^{2}-n w=0$, and $S_{c}=n$. Hence, we could figure out that $\lambda_{a}=0$. In the next, we should discuss what the $\lambda_{c}$ should be.

Since we have set the cross-correlation restrain of each subcode as 1 , and the sum of cross-correlations is $n$, in order to insure $\lambda_{c}$ as 1 , there could not be two subcodes achieve their correlation peek at the same time in a specific code. The theory of Galois Field.

In 1983, Shaar A.A proposed the first practical optical code for OCDMA system, which is named as prime sequence [8]. In the construction of prime sequence, the theory of Galois Field is applied. In this paper, we will apply a method which is similar with the construction method of prime codes in OCCs' construction. By this way, we could maintain the cross-correlation constraint as 1 , that is $\lambda_{c}=1$.

With the rules above, we will construct a new family of optical codes with the ideal correlation properties called optical complementary codes (OCCs).

\section{Construction Method of OCCs}

In this section, the instructions for code construction will be proposed, and the theory of Galois Field will be used here. Since the detail of Galois Field theory does not matter a lot here, we will not discuss this issue in this paper. For more details of Galois Field theory, readers could refer to [8] and [2].

Firstly, let us define the length of subcode as $p$ where $p$ is a prime number and $p>2 . G F(p)$ denotes a Galois Field function. The integer $i$ denotes the index of code in a code set and $j$ is the index of the subcode of a specific code, $i, j \in G F(p)$.

$$
a_{i}^{j}=i \otimes_{p} j
$$

where $\otimes_{p}$ is the production of $i$ and $j$ with modulo $p$, and the value of $a_{i}^{j}$ denotes the position of ' 1 ' in subcode $j$ of code $i$ and it begins with 0 . Each code is distributed for one user. If reformed into matrix, it could be described as $(p \times p, p, 0,1)$, and each code could be

$$
\boldsymbol{a}_{i}=\left[\begin{array}{c}
a_{i}^{0} \\
a_{i}^{1} \\
\vdots \\
a_{i}^{p-1}
\end{array}\right]
$$

Taking $p=7$ as an example to describe the construction method in details, $G F(7)$ is a Galois Field by order 7. So, we could figure out that $G F(7)=\{0,1,2,3,4,5,6\}$. By the equation (11), we could figure out all the $a_{i}^{j}$ in the Optical Complementary Codes set as Table 1. $\boldsymbol{a}_{i}$ is code in this code set, and $a_{i}^{j}$ is one of the sub-codes in code $\boldsymbol{a}_{i}$

With the instructions above, we could get a binary code easily. Taking $a_{4}^{3}=5$ as an example. $a_{4}^{3}$ means 
the forth subcode in the fifth code in code set and $a_{4}^{3}=5$ means this code could be presented as $\left[\begin{array}{lllllll}0 & 0 & 0 & 0 & 0 & 1 & 0\end{array}\right]$. By Table 1, the fifth code $\boldsymbol{a}_{4}$ is

$$
\boldsymbol{a}_{4}=\left[\begin{array}{lllllll}
1 & 0 & 0 & 0 & 0 & 0 & 0 \\
0 & 0 & 0 & 0 & 1 & 0 & 0 \\
0 & 1 & 0 & 0 & 0 & 0 & 0 \\
0 & 0 & 0 & 0 & 0 & 1 & 0 \\
0 & 0 & 1 & 0 & 0 & 0 & 0 \\
0 & 0 & 0 & 0 & 0 & 0 & 1 \\
0 & 0 & 0 & 1 & 0 & 0 & 0
\end{array}\right]
$$

For any prime number $p$, we could get a code set with the code length $p^{2}$ and code weight $p$. The prime number could be determined by specific condition. Compared with 2-D OOC, OCCs shares lower crosscorrelation interferences and OCCs have terminated the auto-correlation interference. Furthermore, the construction method of OCCs is very simple and the codes could be applied easily.

\section{Correlation Properties and Capacities of OCCs}

In this section, the auto-correlation and cross-correlation properties would be discussed with an example as OOCs $(7 \times 7,7,0,1)$. The capacity of codes would be compared with 2-D OOC since 2-D OOC is an important sample of conventional two dimensional optical codes [2]

\subsection{Correlation Properties of OCCs}

With Table 1, we could get the whole optical comple- mentary code set on prime number 7. Taking any two codes, $a_{1}$ and $a_{1}$ for example, we could get the codes as follows,

$$
a_{1}=\left[\begin{array}{lllllll}
1 & 0 & 0 & 0 & 0 & 0 & 0 \\
0 & 1 & 0 & 0 & 0 & 0 & 0 \\
0 & 0 & 1 & 0 & 0 & 0 & 0 \\
0 & 0 & 0 & 1 & 0 & 0 & 0 \\
0 & 0 & 0 & 0 & 1 & 0 & 0 \\
0 & 0 & 0 & 0 & 0 & 1 & 0 \\
0 & 0 & 0 & 0 & 0 & 0 & 1
\end{array}\right] \quad a_{2}=\left[\begin{array}{lllllll}
1 & 0 & 0 & 0 & 0 & 0 & 0 \\
0 & 0 & 1 & 0 & 0 & 0 & 0 \\
0 & 0 & 0 & 0 & 1 & 0 & 0 \\
0 & 0 & 0 & 0 & 0 & 0 & 1 \\
0 & 1 & 0 & 0 & 0 & 0 & 0 \\
0 & 0 & 0 & 1 & 0 & 0 & 0 \\
0 & 0 & 0 & 0 & 0 & 1 & 0
\end{array}\right]
$$

So, the correlation curves are presented at Figure 1. In Figure 1, we could figure out that the auto-correlation peek is 7 and the auto-correlation interferences are 0 . The red dashed with a cross presents the cross-correlation interferences and it is can be found in the figure that all the cross-correlation values are 1 .

Table 1. The Optical Complementary Codes with $p=7$.

\begin{tabular}{cccccccc}
\hline$a_{i}^{j}$ & $j=0$ & $j=1$ & $j=2$ & $j=3$ & $j=4$ & $j=5$ & $j=6$ \\
\hline$i=0$ & 0 & 0 & 0 & 0 & 0 & 0 & 1 \\
$i=1$ & 0 & 1 & 2 & 3 & 4 & 5 & 6 \\
$i=2$ & 0 & 2 & 4 & 6 & 1 & 3 & 5 \\
$i=3$ & 0 & 3 & 6 & 2 & 5 & 1 & 4 \\
$i=4$ & 0 & 4 & 1 & 5 & 2 & 6 & 3 \\
$i=5$ & 0 & 5 & 3 & 1 & 6 & 4 & 2 \\
$i=6$ & 0 & 6 & 5 & 4 & 3 & 2 & 1 \\
\hline
\end{tabular}

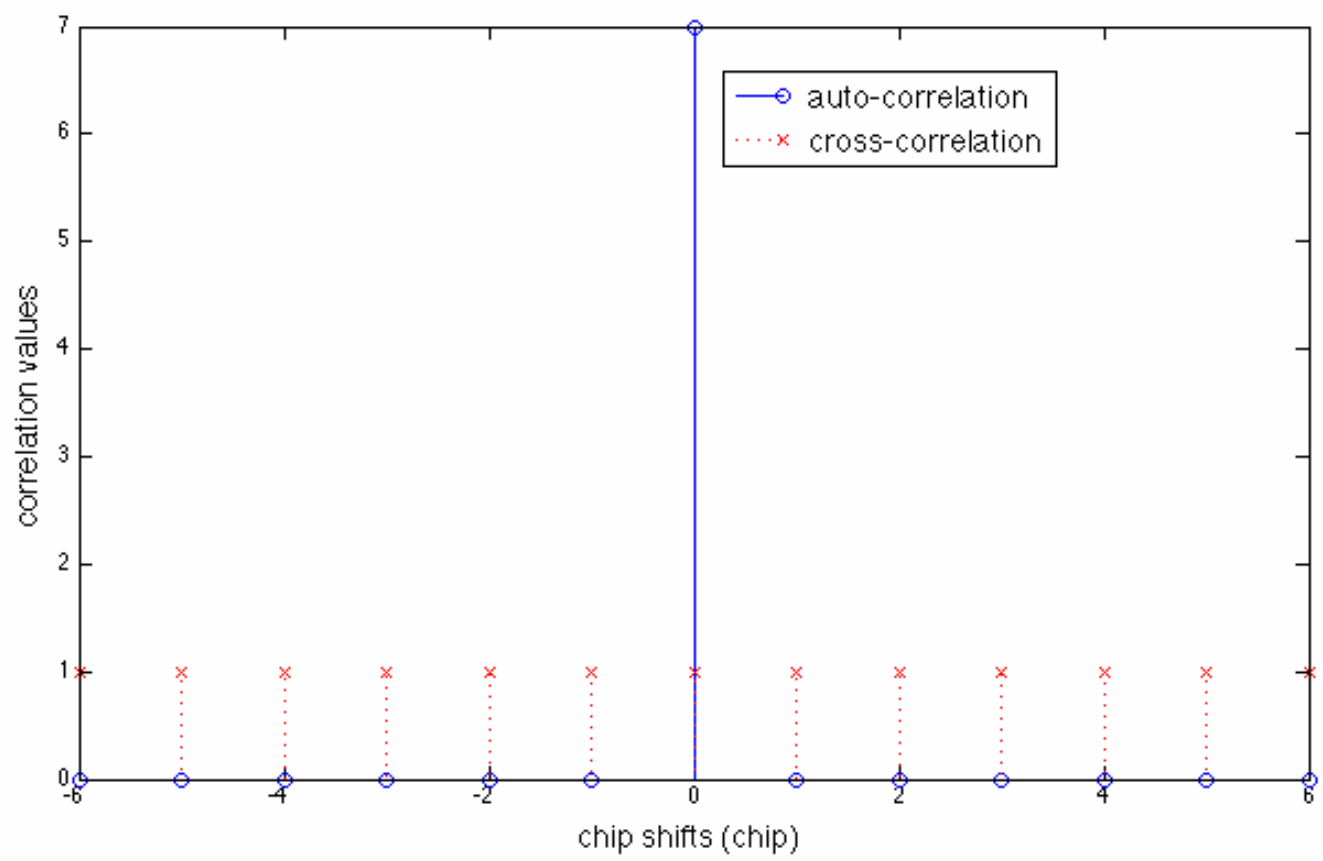

Figure 1. Correlation properties of OCC $a_{1}$ and $a_{2}$. 
For 2-D OOCs, whose auto-correlation side lobes are not zeros, will have to deal with the interference in asynchronous conditions. The cross-correlation of OCCs is the same with 2-D OOCs but more predictable.

\subsection{Capacity of OCCs}

The capacity of optical codes are described with the equation $\Phi\left(n \times m, w, \lambda_{a}, \lambda_{c}\right)$.

According to [2]

$$
\Phi_{2 \mathrm{D}-\mathrm{OOC}}(n \times m, w, \lambda) \leq\left\lfloor\frac{n(n m-1)}{w(w-1)}\right\rfloor
$$

where $\lfloor\cdot\rfloor$ denotes the round down operation.

For the 2-D OOC $(p \times p, p, 1)$, the capacity of it is

$$
\Phi_{\text {2D-ooc }}(p \times p, p, 1) \leq p+1
$$

When it comes to OCCS, for any prime number $p$, with the instructions proposed in section III, we could find $p$ codes for the OCC code set and each code is distributed to one user. Basically, the capacity of OCCs is $p$, which is $\Phi_{\mathrm{occ}}(p \times p, p, 1)=p$. Hence, the capacity of OCCs is similar with OOCs, especially when the prime code is large enough.

Besides, the code length of 2-D OOCs is longer than that of OCCs generally, which requires higher frequency encoders and reduces the spectrum efficiency.

\section{Conclusions}

This paper proposes a new family of optical signature codes for OCDMA system named as Optical Complementary Codes (OCC). The auto-correlation constraints of these codes is 0 and the cross-correlation constrains is 1 , and this optical correlation property is the best that a binary optical code set could reach. Meanwhile, the ca- pacity of OCCs is almost equal to that of 2-D OCCs almost. The construction of these codes adopted the ideas of complementary codes and the construction of each subcode is similar with prime sequence. With the simple instructions, the codes could be built easily, which makes the application of these codes very simple and practical.

\section{REFERENCES}

[1] K. Fouli and M. Maier, "OCDMA and Optical Coding: Principles, Applications, and Challenges,” IEEE Communications Magazine, Vol. 45, No. 8, 2007, pp. 27-34. doi:10.1109/MCOM.2007.4290311

[2] H. Ghafouri-Shiraz and M. M. Karbassian, "Optical CDMA Networks: Principles, Analysis and Applications,” John Wiley \& Sons, 2012. doi:10.1002/9781119941330

[3] Marcel J. E. Golay, "Multislit spectroscopy,” Journal of the Optical Society of America, Vol. 39, 1949, pp. 437-444. doi:10.1364/JOSA.39.000437

[4] Marcel. J. E. Golay, “Complementary Series,” IRE Transactions on Information Theory, 1961(Aprial), pp. 82-87.

[5] H. H. Chen, J.-F. Yeh and N. Suehiro, “A Multicarrier CDMA Architecture Based on Orthogonal Complementary Codes for New Generations of Wideband Wireless Communications,” IEEE Communications Magazine, Vol. 39, No. 10, 2001, pp. 126-135. doi:10.1109/35.956124

[6] L. Xu, I. Glesk, V. Baby, etc., "Multiple Access Interference (MAI) Noise Reduction in a 2D Optical CDMA System Using Ultrafast Optical Thresholding,” Lasers and Electro-Optics Society, 2004, Vol. 2, pp. 591-592.

[7] S. A. Khan and J. Bajcsy, "Chip-Asynchronous Binary Optical CDMA: An Optimum Signaling Scheme for Random Delays,” IEEE Photonics Journal, 2013, Vol. 5, No. 2, Aricle\# 7200408.

[8] A. A. Shaar and P. A. Davids, "Prime Sequences: Quasioptimal Sequences for or Channel Code Division Multiplexing,” Electronics Letters, Vol. 9, No. 21, 1983, pp. 888-889. doi:10.1049/el:19830605 